\title{
Le caractère dramatique des épigrammes de la Croix et de la Crucifixion du Christ
}

Anastasios Kantaras

(Aristotle University of Thessaloniki)

\begin{abstract}
Scholar poetry and the case of the epigram in particular has been a literary expression means not only of ideas and life attitudes but also of religious sentiment and profound religious faith. Delving into the epigrams of the second category and in particular, the epigrams dealing with the Cross and the Crucifixion, our attention will be focused on the tropes employed in these epigrams. In short, we will examine those tropes used by byzantine epigram-writers - anonymous or not - through a range of epigrams such as direct and indirect questions, exclamations and rhetoric questions as well as the influencing factors, the aims and objectives and finally, whether the use of these tropes is successful or not.
\end{abstract}

\section{Keywords}

Byzantium; Byzantine scholar poetry; Byzantine epigram; cross; crucifixion; Christ; dramaticality; dialogue; questions; exclamations 


\section{Introduction ${ }^{1}$}

Avant de procéder à la présentation analytique des épigrammes ${ }^{2}$ qui se réfèrent à la Croix et à la Crucifixion du Christ, je voudrais me pencher sur quelques éléments que je considère comme importants afin de comprendre de la meilleure façon possible les épigrammes. Il est donc tout d'abord utile de signaler qu'avec le terme d'épigramme byzantine nous nous trouvons dans le domaine de la poésie byzantine et plus précisément dans notre cas dans les termes de la poésie byzantine savante. ${ }^{3}$ En deux mots il s'agit d'un discours en vers supérieurs et raffinés (et quelquefois peut-être de compréhension plus difficile) écrit pour sa majorité ${ }^{4}$ en vers dodécasyllabiques (pure création byzantine), ${ }^{5}$ tout en transmettant habituellement dans peu de vers des significations profondes. Nous trouvons certaines de ces épigrammes (anonymes ou avec le nom de poètes d'épigrammes connus) éparpillées dans des manuscrits ${ }^{6}$, sur des épitaphes de stèles funéraires ${ }^{7}$ ou encore sur des peintures murales d'églises ${ }^{8}$, écrites sur des icônes portables, gravées sur des objets sacrés, ${ }^{9}$ tels que des plateaux, des calices, des couvertures d'évangiles et autres, éventuellement des broderies sur des tissus sacrés (nappes d'autel sacré, bannières), ou encore sur des créations plus composites de l'artisanat byzantin comme par exemple des croix en pendentifs, des petits ou grands boitiers de croix et des reliquaires, ${ }^{10}$ tous témoins d'un art raffiné et de haut niveau qui suscite jusqu'à nos jours encore notre admiration. ${ }^{11}$

1 Mon doctorat ayant pour thème la découverte, la collecte et le commentaire des épigrammes qui se réfèrent à la Croix et à la Crucifixion du Christ, j'ai pris la décision d'en présenter quelques-unes en concentrant notre attention sur le caractère dramatique qu'elles peuvent contenir, en essayant de les comprendre, de les interpréter et d'y justifier leur présence.

2 Toutes les traductions des épigrammes de l'article en question sont de l'auteur même de cet l'article.

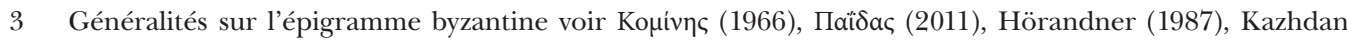

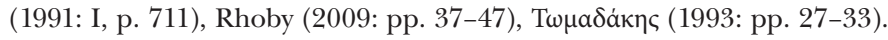

4 Parmi les épigrammes byzantines se trouvent bien sûr d'autres sortes de mètres comme l'hexamètre, le distique élégiaque et le vers de quinze syllabes. Voir Lauxtermann (1999). Mais dans leur majorité les auteurs d'épigrammes byzantins ont surtout fait usage du vers dodécasyllabique - comme le stipule précisément Marc Lauxtermann dans certaines remarques sur les épigrammes de Georges Pisidis, utilisant le terme de «vitium Byzantium» (voir sur ce point Lauxtermann [2003b: p. 182])- et très rarement d'autres genres de vers. Voir Lauxtermann (2003a: p.31), Rhoby (2009: p. 38, p. 62), Rhoby (2010: pp. 40-41), Rhoby (2014: pp. 83-90).

5 Georges Pisidis est considéré comme l'introducteur de l'alexandrin byzantin (dodécasyllabe vers) qui résulte de l'évolution du trimètre iambique antique (VII' siècle). Voir Пaîßac (2011: p. 41). Pour l'alexandrin byzantin voir Maas (1903).

6 Voir Rhoby (2018).

7 Voir Rhoby (2014), Rhoby (2011), Lauxtermann (2003a: pp. 213-240), Brooks (2006).

8 Voir Rhoby (2009).

9 Voir Rhoby (2010).

10 L'habitude très ancienne de l'épigraphe d'hommage ou d'anathème en vers gravée sur des objets se rencontre à l'époque byzantine, en particulier à partir de Constantin VII Porphyrogénète et après, avec l'exemple éminent et le plus représentatif la Staurothèque de Limbourg. Voir à ce sujet Hörandner (1989: p. 150) et Koder (1989).

11 En ce qui concerne la relation de l'épigramme avec la vénération dans l'art byzantin, particulièrement l'art byzantin tardif, voir Drpić (2016). 
Pour ce qui est de leur composition, leurs créateurs, qui sans aucun doute ont lu et connaissent très bien les textes de leurs ancêtres, c'est-à-dire les œuvres des écrivains classiques, poussés par une inquiétude très profondément métaphysique et une croyance en Dieu font un usage fréquent de ces moyens d'expression (termes) si habituels aux écrivains de leurs modèles. En deux mots, il est possible de trouver de telles influences tant dans la technique que dans la langue, sans laisser en même temps tomber dans l'oubli la force pénétrante de l'art rhétorique dans tous les styles et genres de la littérature byzantine, incluant également poésie byzantine et épigramme sacrée. ${ }^{12}$

\section{Dialogue}

En étudiant les épigrammes pour la croix et la crucifixion du Christ, ${ }^{13}$ et en accordant une plus grande attention au caractère dramatique que celles-ci comportent, nous pouvons constater de la part des poètes d'épigrammes, l'usage de l'art du dialogue. Nous rencontrons ainsi une forme de question-réponse entre des figures tantôt abstraites tantôt plus concrètes.

Pour parler de manière plus précise, nous rencontrons des épigrammes qui se réfèrent à des représentations antérieures de la croix dans l'Ancien Testament utilisant le dialogue dans le but d'exposer le fait en question et pour souligner son analogie et sa relation avec celui de la crucifixion du Christ. Nous avons ainsi une épigramme chronologiquement

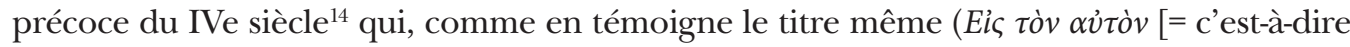

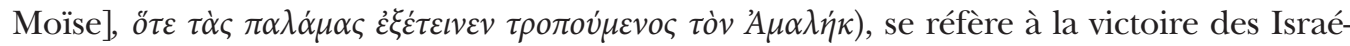
lites dans leur bataille contre le peuple des Amalécites ${ }^{15}$ grâce aux bras étendus de Moïse ${ }^{16}$ en forme d'oraison de la Croix pendant sa prière à Dieu, faisant ainsi de la position des bras de Moïse une norme de la croix ${ }^{17}$ et par extension de Moïse-même la représentation

12 Pour la théorie de la Rhétorique et de sa fonction dans la production littéraire et dans la vie spirituelle de

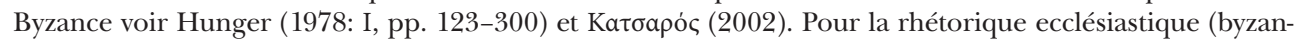
tine) voir Koúkoupa (2011³: pp. 139-280).

13 Il est évident qu'aussi bien la Croix que la Crucifixion du Christ constituaient des thèmes chers aux auteurs d'épigrammes. Je mentionne de manière indicative quelques études sur ce sujet: Frolow (1961), Hörandner (1990), Hörandner (2007), Lauxtermann (2003a: pp. 342-246), Lauxtermann (1994: pp. 3844).

14 Il s'agit de Grégoire de Nazianze, dit Grégoire le Théologien. Pour sa vie et ses œuvres voir Kazhdan (1991: II, pp. 880-822) et Maptívos (1964: 4, pp.708-729).

15 Selon le Livre de l'Exode (Ex. 17,8-16) Moïse incite Jésus de Navé à combattre les Amalécites tandis qu'il surveillerait lui-même l'évolution de la bataille d'un endroit élevé, priant Dieu pour une issue heureuse et en ayant à ses côtés son frère Aaron, et son beau-frère Or, du côté de sa sœur Marie. Là, donc, sur la hauteur, tant que Moïse avait les bras levés tenant la verge de Dieu (Ex. 17,9), les Israélites gagnaient mais quand il les baissait, c'étaient les Amalécites qui avaient le dessus. Alors Aaron et Or aidèrent Moïse à toujours maintenir les bras levés jusqu'à la victoire finale des Israélites $(E x .17,12)$.

16 Je souligne que la position des bras levés de Moïse en forme d'oraison (croix) ne se rencontre pas seulement dans ce fait mais dans d'autres encore comme par exemple lorsqu'il a prié et qu'une obscurité profonde a recouvert toute la terre d'Egypte durant trois jours (Ex. 10,21-23).

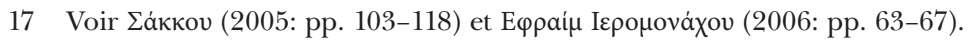


du Christ. ${ }^{18}$ Cette épigramme précise est constituée de deux vers dont le premier est la question de l'écrivain et donc celle de chaque fidèle pieux à Moïse même, alors que le second en constitue la réponse:

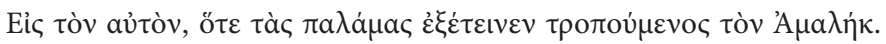

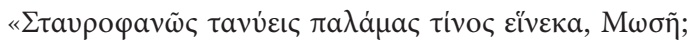

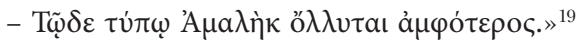

\section{Traduction:}

A Moïse lui-même, quand il a étendu les bras, a fait fuir l'Amalek. «Pour quelles raisons étends-tu les bras en forme de croix, Moïse?

- De cette manière je fais disparaître les deux Amalek.»

Un dialogue de cette nature et de plus avec une figure sacrée de l'Ancien Testament, telle que Moïse, montre à mon avis, d'une part, la profonde connaissance du poète d'épigramme des textes sacrés et, d'autre part, la parfaite compétence de l'usage de l'élément de dialogue pour une version dramatisée du message de l'épigraphiste qui n'est autre qu'une préfiguration de la croix et de la crucifixion de Jésus et la force qui en découle afin de vaincre le mal quelle que soit la forme qu'il puisse prendre.

L'élément de dialogue est cependant rencontré dans deux épigrammes de Théodore Prodrome (XIIe siècle). ${ }^{20}$ L'une traite du cas du serpent de cuivre, lequel sur ordre de Dieu a été réalisé et élevé sur du bois par Moïse et placé sur une hauteur afin que les Israélites qui étaient victimes du poison des serpents venimeux du désert ne meurent pas s'ils dirigeaient leur regard vers celui qui leur apportait la délivrance du mal et leur redonnait la santé. ${ }^{21} \mathrm{~A}$ travers ce cas, ${ }^{22}$ l'épigraphiste percevait donc le serpent de cuivre posé sur du bois comme la préfiguration de la crucifixion du Christ sur le bois de la croix. Plus précisément le serpent ${ }^{23}$ est la forme du Christ et le bois sur lequel le serpent de

18 Nous nous devons de souligner bien sûr que Amalek constitue l'expression de Satan et du mal, tandis que dans cette victoire de Jésus de Navé sur l'Amalek nous voyons au travers de la croix la victoire du Christ

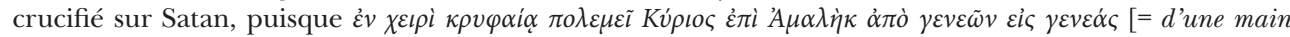
cachée le Seigneur combat Amalek de génération en génération] $(E x .17,16)$. De surcroit l'idée selon laquelle le mont sur lequel Moïse est monté et s'est tenu debout représente le Golgotha et que finalement les deux accompagnateurs de Moïse, Aaron et Or figurent les deux voleurs puisque comme eux ils se tiennent à gauche et à droite de Moïse à la place du Christ.

19 Beckby (1964²: I, p. 152 [nº0]). Comme chacun peut le constater, l'épigramme n'est pas écrite en vers dodécasyllabique typiquement byzantin, qui est adopté principalement à partir du VIIe siècle et après, mais en distique élégiaque puisqu'il s'agit d'épigramme de la période byzantine précoce (IVe siècle) qui répercute encore l'influence des mètres de la poésie antique. Le distique élégiaque (mètre des épigrammes antiques) disparait essentiellement à partir de l'an 600 et après. Voir Lauxtermann (2003a: p. 31).

20 Pour sa vie et ses œuvres voir Kazhdan (1991: III, pp. 1726-1727).

21 Num. 21,8.

22 Pour l'exposition relative à ce fait voir इáккоv (2005: pp. 139-144), alors que pour la préfiguration précise

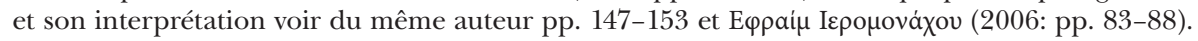

23 Pour la signification du mot serpent avec lequel il répond dans la Bible voir इákкov (2005: pp. 147-148). Aussi voir Tıаvvapác (2009: pp. 63-65). 
cuivre a été placé la forme de la croix. Quel peut être ensuite le moyen de présentation approprié de cette préfiguration, de ce dialogue, cette fois-ci entre deux figures que nous pouvons imaginer être, d'un côté l'auteur de l'épigramme et de l'autre son lecteur? Un dialogue qui se trouve et se limite donc seulement à quatre vers:

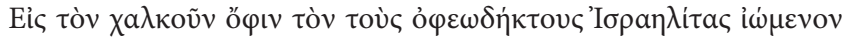

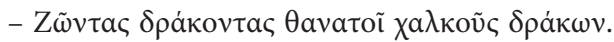

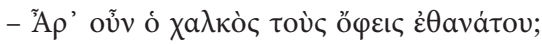

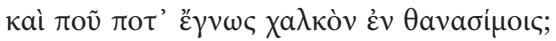

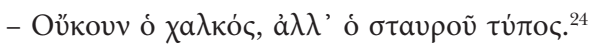

\section{Traduction:}

$\mathrm{Au}$ serpent de cuivre qui guérit les Israélites mordus.

- Le serpent de cuivre provoque la mort à des serpents vivants.

- Le cuivre tuait-il donc peut-être les serpents?

Et où, et quand as-tu appris que le cuivre est mortel?

- Non pas, bien sûr le cuivre mais la forme de la croix

Le deuxième quatrain, épigramme de Théodore Prodrome également, qui est écrite sous forme de dialogue, se présente ainsi:

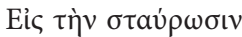

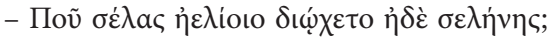

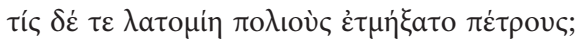

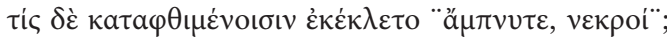

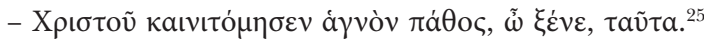

\section{Traduction:}
A la crucifixion
- Où a disparu la vive lumière du soleil et de la lune?
quelle carrière a fendu en deux les pierres grises?
qui a crié aux morts «respirez morts»?
- C'est la Sainte Passion du Christ qui a fait cela, étranger.

24 Papagiannis (1997: II, pp. 81-82 [n 73a]).

25 Papagiannis (1997: II, pp. 239-240 [n²29b]). Pour le mètre de l'épigramme en question, qui n'est pas écrit en vers dodécasyllabique byzantin, je cite le point de vue de Lauxtermann sur les mètres des épigrammes byzantines: «The usual metre is the dodecasyllable, either in its prosodic or unprosodic form. Almost all Byzantine epigrams make use of the dodecasyllable, with a few classicizing exceptions in hexameters or elegiacs.» Voir Lauxtermann (2003a: p. 31). 
En lisant l'épigramme, nous pouvons constater que le contenu est en relation avec les événements suivant la mort du Christ crucifié, pendant lesquels le soleil s'est caché, qu'une obscurité profonde s'est répandue et qu'un grand séisme a suivi et qui a détruit des constructions, ${ }^{26}$ parmi lesquelles le Temple de Solomon. Pour la présentation de son effondrement sur la terre comme réaction de la nature au spectacle terrible et effrayant de la crucifixion du Dieu des hommes, le créateur de l'épigramme choisit l'art du dialogue entre lui-même et chaque lecteur de cette épigramme-là ( $\tilde{\omega} \xi \xi \dot{\varepsilon} v$ [O étranger]: vers 4), de façon à souligner davantage la signification et l'importance du sacrifice du Fils de Dieu.

Dans les deux épigrammes suivantes nous voyons un dialogue prendre place entre leur créateur et par extension des lecteurs, et avec le Christ lui-même. Dans la première épigramme, œuvre d'un auteur inconnu du Xe siècle laquelle est aussi constituée de huit vers, nous avons le changement des personnes en dialogue, commençant par l'interroga-

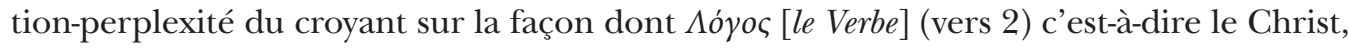
assis au côté de son Père dans le ciel est descendu sur terre à travers une кó pure] (vers 1-2), c'est-à-dire la Sainte Vierge mère de Jésus sans altérer ni changer sa Nature. Puis s'ensuit la réponse de la bouche du Christ lui-même, qui explique comment cet événement miraculeux est expliqué par les paroles de tous les prophètes, alors qu'à la fin arrive le fidèle pour confirmer la déclaration de Jésus Christ qu'effectivement est arrivé le moment de l'exaucement des prophéties qui précédaient :

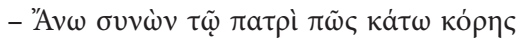

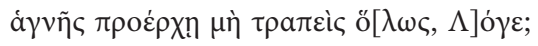

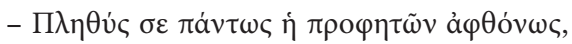

$\kappa a \theta \dot{\omega}[\sigma \pi \varepsilon] \rho \dot{\varepsilon} \beta \rho o ́ v \tau \eta \sigma \varepsilon v, \dot{\varepsilon} \kappa \delta เ \delta \alpha \sigma \kappa \varepsilon \dot{\tau} \omega$.

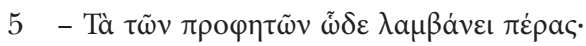

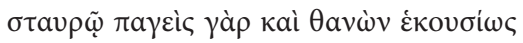

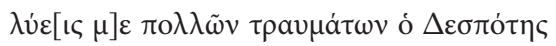

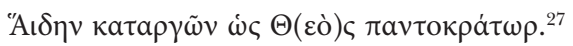

Traduction:

- Puisque tu es en haut avec ton père, comment sors-tu ici-bas d'une fille

pure sans avoir du tout été altéré, Verbe?

La foule des prophètes te l'expliquera assurément

avec ses mots retentissants.

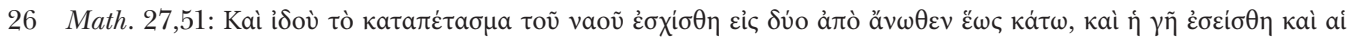

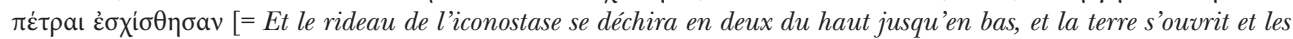
pierres se fendirent]. Pour la réaction de la nature qui par peur devant la criminalité de l'homme exprime de

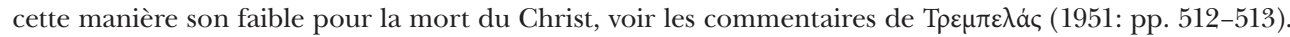

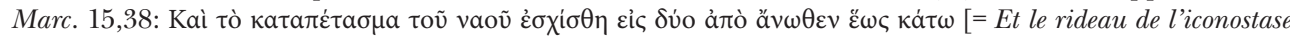
se déchira en deux de haut en bas].

27 Epigramme sur diptyque en ivoire. Voir Rhoby (2010: pp. 329-331 [n . El22] et p. 524 [im. 93]), Kiourtzian (2005: p. 236 et 234 [im. 1]). 
5 - Les paroles des prophètes sont à ce moment accomplies parce que crucifié sur la croix et mort volontairement toi, Souverain suprême, tu me délivres de nombreuses plaies, abolissant l'Enfer en tant que Dieu tout puissant.

La deuxième épigramme en question, œuvre de Nicolas d' Otrante ${ }^{28}$ (XIIe-XIIIe siècle) est formé de six vers dont les quatre premiers appartiennent au lecteur-croyant qui s'adresse au Christ, cette fois non pas sous la forme de question mais sous la forme d'une simple déclaration affirmative relative au « repos » du Christ, en tant que souverain, sur le bois de la croix fait à partir du bois de trois arbres,${ }^{29}$ le comparant au sommeil dont il se réveillera bientôt, et révélant de cette manière Sa Résurrection attendue. Les deux derniers vers (vers cinq et six) constituent la confirmation du Fils de Dieu lui-même que malgré le fait qu'il « dorme » sur le bois de la croix, Son cour reste cependant vigilant, anéantissant les chaines de l'Enfer et sauvant ainsi tout le genre humain :

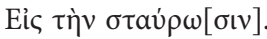

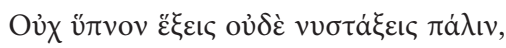

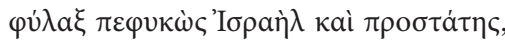

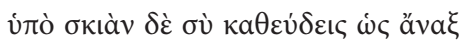

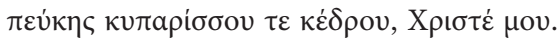

O Xpıбт́́

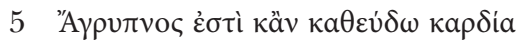

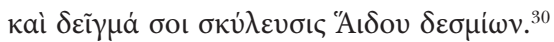

Traduction:

A la crucifixion

Tu n'auras ni sommeil ni n'auras plus jamais envie de dormir, (Toi) qui était le gardien et le protecteur d'Israël, c'est en tant que souverain que Tu reposes à l'ombre du pin, du cyprès et du cèdre, mon Christ.

Le Christ

5 Éveillé est mon cœur même si je dors, Et la preuve pour toi en est la dilapidation des prisonniers de l'Enfer.

Il faut ajouter une mention particulière pour l'épigramme distique du IX ${ }^{\mathrm{e}}$ siècle, œuvre d'un auteur anonyme, selon lequel l'épigraphiste s'adresse cette fois directement à la Sainte Croix en la personnifiant de cette manière. Et ainsi à la question adressée à la Sainte Croix de savoir qui la pare, la Sainte Croix répond que c'est le corps du Christ,

28 Ydroutinos en grec. Pour sa vie et ses œuvres voir Kazhdan (1991: II, pp. 1470-1471).

29 Il s'agit du dit $\tau \rho \iota \delta \varepsilon v \delta \rho i \alpha$ [= trois bois], puisque le bois de la croix de la crucifixion du Christ était fait de bois de pin, de cyprès et de cèdre.

30 Longo \& Jacob (1980-1982: p. 197 [n 19.7, f. 36 $\left.6^{\mathrm{r}}\right]$ ). 
qui y a été cloué et qui lui donne à la fois une nouvelle grâce et beauté puisqu'il ne s'agit pas de n'importe quel corps mais de celui du Fils de Dieu:

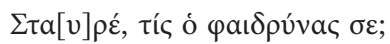

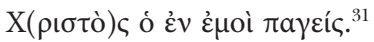

\section{Traduction:}

Sainte Croix, qui t'a parée?

Le Christ qui y a été cloué.

Pour fermer la parenthèse des exemples des épigrammes de la croix et de la crucifixion qui utilisent la technique du dialogue, je voudrais en souligner encore une, celui de Manuel Philès, homme de lettres byzantin du XIII ${ }^{\mathrm{e}}-\mathrm{XIV}{ }^{\mathrm{e}}$ siècle, ${ }^{32}$ qui fait une allusion particulière à la représentation du corps du Christ sur la croix. Plus précisément l'épigraphiste demande au peintre pour quelle raison alors qu'il représente le Christ crucifié sur la croix, il n'accentue pas clairement dans son dessin Sa nature divine. Le peintre répond que puisqu'on lui a appris à représenter les corps de façon globale, dans le cas du corps du Christ crucifié, ses compétences artistiques n'étaient alors pas suffisantes pour montrer tout le sublime que ce corps divin possède. Cette épigramme de quatre vers est coupée par son auteur exactement à la moitié, avec la question dans les deux premiers vers et avec la réponse dans les deux derniers vers, donnant de cette manière une symétrie à l'épigramme:

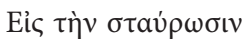

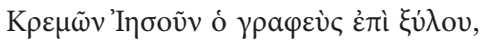

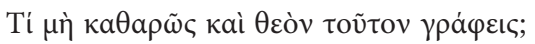

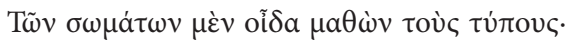

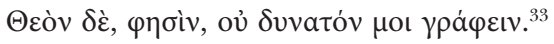

Traduction:

A la crucifixion

En représentant, peintre, le Christ accroché sur le bois

31 Epigramme sur fresque de narthex d'église en Turquie (Yılanlı Kilisesi) concernant le dialogue entre le visiteur de l'église et l'image (de la croix). Rhoby (2009: pp. 294-295 [ $\mathrm{n}^{\circ}$ 202] et p. 489 [im. 74]), Rott (1908: p. 273), Paul (2008: p. 72 [n 21]), Thierry (2002: p. 155 [Dessin 59]), Thierry \& Thierry (1963: p. 91), Schiemenz (1987-1989: p. 56), Hörandner (2002: pp. 157-158). Concernant la métrique, l'épigramme est constituée de deux vers de huit syllabes lesquels ont un rapport avec le vers de quinze syllabes vu que le vers de huit syllabes peut être considéré comme la moitié du vers de quinze syllabes. Voir Rhoby (2009: p. 295). D'ailleurs l'utilisation rare du vers de quinze syllabes par les auteurs d'épigrammes byzantins commence au XIe siècle. Voir Lauxtermann (2003a: p. 31). Pour le vers byzantin de huit syllabes voir Lauxtermann (1999: pp. 45-49).

32 Pour sa vie et ses œuvres voir Kazhdan (1991: III, p. 1651).

33 Miller (1855-1857 = 1967: I, p. 97 [n CXCVII]). 
comment ne l'as-tu pas dessiné clairement comme Dieu?

On m'a appris et je sais comment représenter les corps

Mais celui de Dieu, dit-il, il m'est impossible de le peindre.

A ce point j'aimerais souligner le fait que le recours à l'utilisation du dialogue dans la poésie byzantine n'est pas quelque chose d'inhabituel, bien au contraire, nous le rencontrons en dehors du domaine des épigrammes (bien sûr avec pour thème pas seulement celui de la croix et de la crucifixion du Seigneur), mais aussi dans la poésie ecclésiastique et dans les recueils d'hymnes avec pour forme principale celle de Romanos le Mélode (VIe siècle), ${ }^{34}$ où le dialogue tant intérieur qu'extérieur, ${ }^{35}$ donne de la vigueur en montrant les conflits de l'âme des élus.

\section{Questions rhétoriques}

Dans les épigrammes néanmoins concernant la croix et la crucifixion, nous pourrions être amenés à rencontrer des questions simples sans que des réponses soient données, c'est-à-dire des questions rhétoriques étant donné que le lecteur connait d'avance la réponse ou a la possibilité de la supposer. Ces questions ont pour but principalement de donner un ton encore plus dramatique à l'épigramme de façon à déclarer l'affliction émotionnelle tout d'abord de l'épigraphiste et par la suite de chaque lecteur à propos du terrible spectacle de la crucifixion du Fils de Dieu.

Ainsi donc le poète s'étonne de voir le Christ crucifié sur l'instrument de châtiment des criminels, c'est-à-dire la croix :

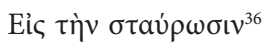

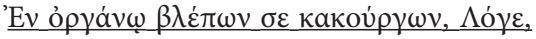

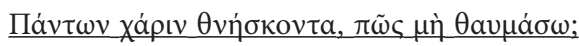

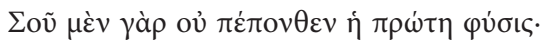

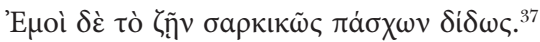

Traduction:

A la crucifixion

Te voyant, Verbe, sur l'instrument des criminels (= sur la croix)

mourir pour la grâce de tous, comment ne pas s'interroger?

Ta première nature n'a évidemment pas souffert

mais avec Ta passion Tu m'offres la vie dans le corps.

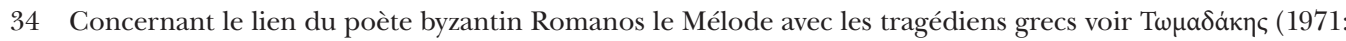
pp. 130-152) et Iorga (2017: pp. 82, 155, note 66).

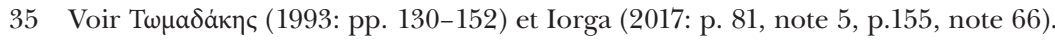

36 Epigramme de Manuel Philès, XIII ${ }^{\mathrm{e}}-\mathrm{XIV}^{\mathrm{e}}$ siècle (1275-1345).

37 Miller (1855-1857 = 1967: I, p. $\left.7\left[\mathrm{n}^{\circ} \mathrm{XVI}\right]\right)$. 
Plus précisément il s'interroge sur le fait que le Christ ait étendu Ses bras sur le bois de la croix, endurant les douleurs terribles des clous:

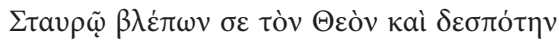

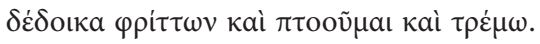

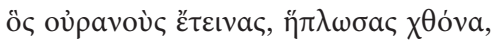

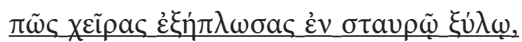

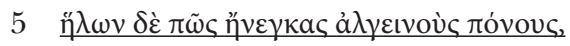

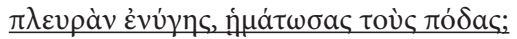

... ${ }^{38}$

Traduction:

Te voyant sur la croix, Dieu et souverain

je ressens de la peur, frémissant et je m'agite et tremble.

(Toi) qui a déployé les cieux (et) a étendu la terre,

comment as-tu étendu les bras sur le bois de la croix,

5 comment as-tu supporté les terribles douleurs des clous,

(comment) ton flan piqué (le tien), tes pieds en sang (les tiens)? $\cdots$

Et même entre des voleurs, comme s'il était Lui-même un des leurs aussi:

Eiç $\Sigma \tau \alpha u ́ \rho \omega \sigma i v$

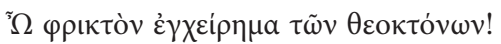

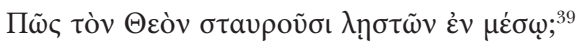

Traduction:

A la crucifixion

Ô terrible action des tueurs de Dieu!

Comment ont-ils crucifié Dieu entre des voleurs?

Enfin pour donner un plus grand caractère dramatique, une intensité émotionnelle et une immédiateté, l'épigraphiste n’hésite pas à mettre le Christ crucifié lui-même à s'adresser au lecteur et dans un sens plus large à n'importe quel homme avec une question rhétorique, peut-être un embarras, soulignant le sacrifice ultime qu'il fait pour la rédemption du genre humain:

38 Epigramme du XII ${ }^{\mathrm{e}}$ siècle totalement avec 11 vers, écrit par Grégoire Pardo, Métropolite de Corinthe. Hunger (1982: p. 642 [n VI]). Pour la vie et ses œuvres voir Kazhdan (1991: III, p. 1587).

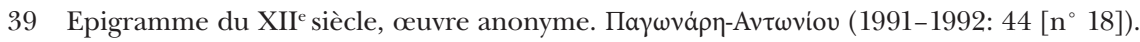




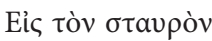

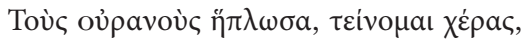

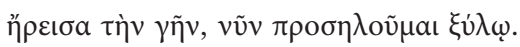

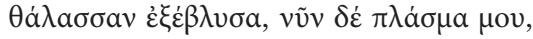

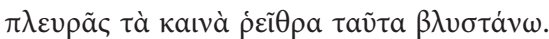

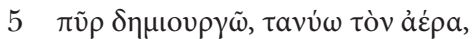

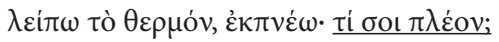

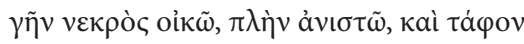

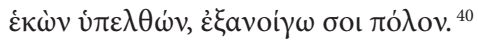

\section{Traduction:}

A la croix

J'ai déployé les cieux, (et maintenant) j'étends les bras

j'ai consolidé la terre, et maintenant je suis cloué sur le bois.

J'ai fait jaillir la mer, mais maintenant, ma créature, de mon flan je fais jaillir les nouveaux courants.

5 Je crée le feu, je répands l'air, j'abandonne la chaleur, je rends l'âme. Pour toi, que faire de plus? Je réside mort sur la terre, et malgré cela je ressuscite, et dans la tombe descendu de mon propre gré, j'ouvre tout grand le ciel pour toi.

\section{Exclamations-Interjections.}

Cependant, excepté la technique du dialogue et des questions rhétoriques pour la déclaration du caractère dramatique, nous rencontrons également dans les épigrammes de la croix et de la crucifixion l'usage de certaines exclamations-interjections quelquefois accompagnées par une série de questions pour souligner plus profondément et pour faire une déclaration des émotions de l'épigraphiste comme cela apparait par exemple dans l'épigramme de Jean Mavropous, Métropolite d' Euchaita (XI ${ }^{\mathrm{e}}$ siècle) ${ }^{41}$ pour la crucifixion:

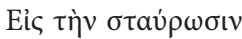

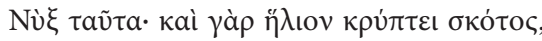

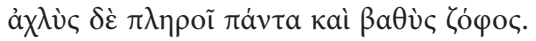

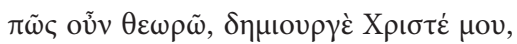

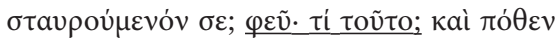

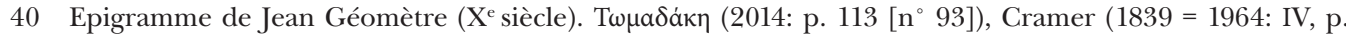
297.9-16), Opstall (2008), Migne (1857-1866: vol. 106, col. 934 A-B[ [६ '] , Joannis Geometrae, carmina varia argument sacri vel historici), Cougny (1890: III, p. 348 [n³45]), Boissonade (1829-1833 = 1962: II, p. 477 [к५']).

41 Pour sa vie et ses ouvres voir Kazhdan (1991: II, p. 1319) et Maptivoc (1965: 7, pp. 4-6). 


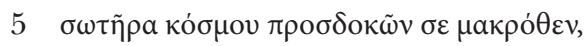

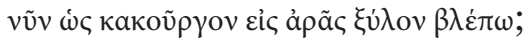
... ${ }^{42}$

\section{Traduction:}

\section{A la crucifixion}

C'est la nuit, puisque l'obscurité recouvre le soleil et qu'un brouillard inonde tout et des ténèbres épaisses.

Comment te voir donc, mon Christ créateur, crucifié ? Malheur, qu'est-ce cela ? Pourquoi

5 t'attendant ici depuis longtemps comme sauveur du monde je te vois maintenant comme un malfaiteur sur le bois de la malédiction?

D’autres fois encore, les exclamations rappellent les tragédies des tragédiens de la Grèce antique puisqu'elles donnent avec l'expression des émotions les plus profondes des épigraphistes devant le terrible spectacle de la crucifixion du Fils de Dieu, et un accent de théâtralité et une vivacité aux vers également. L'épigramme de Nicolas Kalliklès (médecin et homme de lettres du $\mathrm{XI}^{\mathrm{e}}-\mathrm{XII}^{\mathrm{e}}$ siècle $)^{43}$ en constitue un exemple caractéristique dédié au Christ mort crucifié et lié à l'idée de Sa crucifixion avec sommeil:

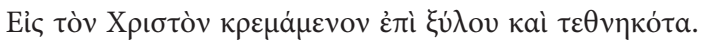

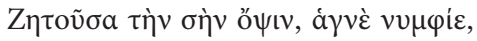

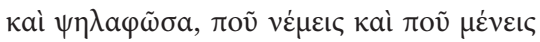

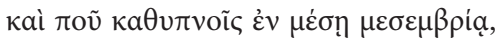

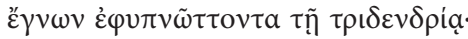

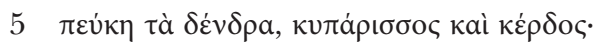

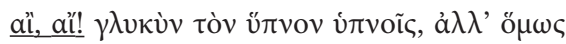

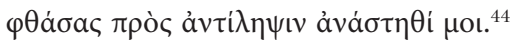

\section{Traduction:}

Pour le Christ mort accroché sur la croix

En quête de ta forme, époux pur,

et cherchant où tu fais paître ton troupeau, où tu habites

et où tu te laisses aller au sommeil en plein midi

j'ai compris que tu dormais sur trois arbres

5 Pin, cyprès et cèdre sont les arbres

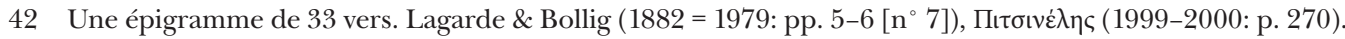

43 Pour sa vie et ses ouvres voir Kazhdan (1991: II, p. 1093).

44 Romano (1980: p. 82 [n 7], p. 135 [traduction italienne] et pp. 168-169 [commentaires]), Frolow (1961: p. 330 [n³38]), Frolow (1941: p. 235), Sternbach (1904: pp. 319-320 et p. 360), Sola (1911: p. 376). 
Même si ton sommeil est doux,

Réveille-toi cependant et viens-moi en aide.

Enfin l'usage d'interjections peut avoir comme but l'expression de l'admiration de l'épigraphiste pour la grandeur de la force spirituelle du Christ, qui s'est sacrifié par une générosité démesurée et un amour extraordinaire pour le salut de l’homme :

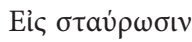

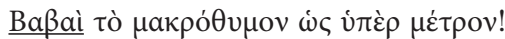

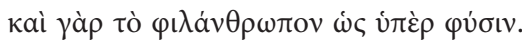

.$^{45}$

Traduction:

A la crucifixion

Quelle générosité magnanime

Et quel amour extraordinaire pour l'homme!

$\cdots$

\section{Conclusions}

Avec la citation ci-dessus et les commentaires de quelques exemples seulement nous pouvons en déduire que sans aucun doute les épigraphistes font un usage admirable et exceptionnel de la langue savante et des exigences rhétoriques, plaçant leurs épigrammes à un niveau élevé et avec une intégrité, non seulement par le soin de leur style mais aussi par la vérité et la force de leur contenu.

Ce qui tient indéniablement pour des aides importantes, ce sont ces techniques qui renforcent et accentuent fortement le caractère dramatique dans ces épigrammes, ces derniers ayant comme but d'une part la mise en avant de la foi profonde de leurs créateurs et d'autre part la révélation de la lutte intérieure de leur monde émotionnel face au terrible et horrible spectacle de la crucifixion de Jésus Christ par les hommes eux-mêmes, techniques comme le dialogue, les questions rhétoriques et les exclamations diverses et les interjections. Avec le choix de ces subterfuges rhétoriques, les auteurs d'épigrammes réussissent d'un côté à attirer l'attention du lecteur-spectateur et de l'autre à provoquer son émotion en le faisant participer par son abattement émotionnel personnel face à la Passion de Jésus Christ.

De cette manière les auteurs de ce genre d'épigramme (comprenant ce thème précis) réussissent à conférer de façon significative l'adoration et le symbolisme (puissant) tant de la croix que de la crucifixion du Christ dans l'âme des croyants.

45 Une epigramme de 12 vers. Miller (1883: p. 44). 


\section{Bibliographie}

Balmelle, C., Chevalier, P., \& Ripoll, G. (Eds.). (2005). Mélanges d'antiquité tardive. Studiola in honorem Noël Duval (Bibliothèque de l'antiquité tardive, 5). Turnhout: Brepols.

Beckby, H. (1964²). Anthologia Graeca: Griechisch-Deutsch (Vols. I-IV). München.

Boissonade, J. Fr. (1829-1833=1962). Anecdota Graeca e codicibus regiis (Vols. I-V). Parisiis: Regium Typographeum (= Hildesheim: Olms).

Brooks, S. (2006). Byzantium: Faith and Power (1261-1557). Perspectives on Late Byzantine Art and Culture (The Metropolitan Museum of Art Symposia). New Haven - London.

Cougny, E. (1890). Epigrammatum Anthologia Palatina cum Planudeis et Appendice Nova. Paris: Firmin-Didot.

Cramer, J. A. (1839=1964). Anecdota Graeca e codd. manuscriptis bibliothecae regiae Parisiensis (Vols. I-IV). Oxonii: Typographeum Academicum (= Hildesheim: Olms).

Drpić, I. (2016). Epigram, Art, And Devotion in Later Byzantium. Washington: University of Washington.

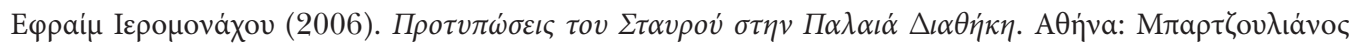
I. H入ías.

Frolow, A. (1941). Deux inscriptions sur des reliquaires byzantins. Revue archéologique $6^{e}$ sér., 18, 233-242.

Frolow, A. (1961). La relique de la vraie croix. Recherches sur le développement d'un culte (Archives de l'Orient chrétien, 7). Paris: Inst. français d'études byzantines.

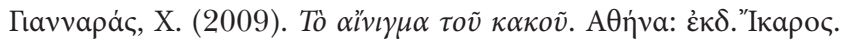

Hörandner, W. (1987). Customs and Beliefs as Reflected in Occasional Poetry. Some Considerations. Byzantinische Forschungen, 12, 235-247.

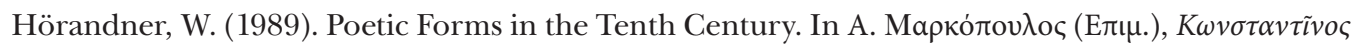

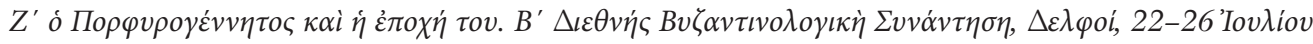

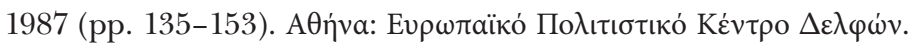

Hörandner, W. (1990). Visuelle Poesie in Byzanz. Versuch einer Bestandsaufnahme. Jahrbuch der Österreichischen Byzantinistik 40, 1-42.

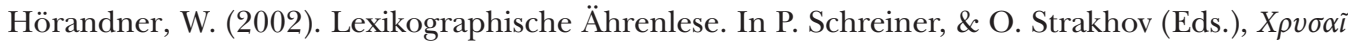

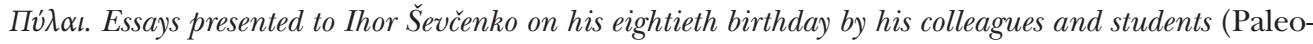
slavica, 10/1-2; Vol. I, pp. 157-158). Cambridge, Mass.

Hörandner, W. (2007). Das byzantinische Epigramm und das heilige Kreuz: einige Beobachtungen zu Motiven und Typen. In B. Ulianich - U. Parente (Eds.), La Croce. Iconografia e interpretazione (secoli I - inizio XVI). Atti del convegno internazionale di studi. (Napoli, 6-11 dicembre 1999). (Vol. III, pp. 107-125). Naples - Rome.

Hörandner, W., \& M. Grünbart (Eds.). (2003). L'épistolographie et la poésie épigrammatique: projets actuels et questions de méthodologie. Actes de la 16e Table ronde organisée par W. Hörandner et M. Grünbart dans le cadre du XXe Congrès international des Études byzantines, Collège de France - Sorbonne, Paris, 19-25 Août 2001. Paris: Dossiers byzantins 3.

Hörandner, W., \& Rhoby, A. (Eds.). (2008). Die kulturhistorische Bedeutung byzantinischer Epigramme. Akten des internationalen Workshops (Wien, 1.-2. Dezember 2006) (Veröffentlichungen zur Byzanzforschung, XIV). Wien: Verlag der Akademie der Wissenschaften. 
Hunger, H. (1978). Die hochsprachliche profane Literatur der Byzantiner (Vols. I-II; Handbuch der

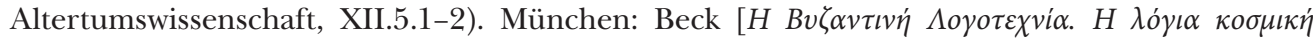

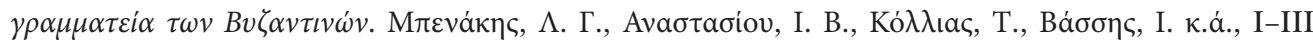
(2008). AӨńva: MIET].

Hunger, H. (1982). Gregorios von Korinth. Epigramme auf die Feste des Dodekaorton. Analecta Bollandiana, 100, 637-651.

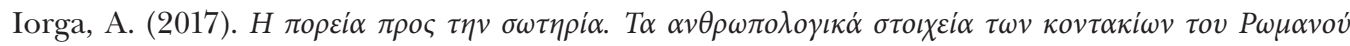

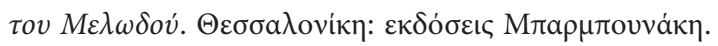

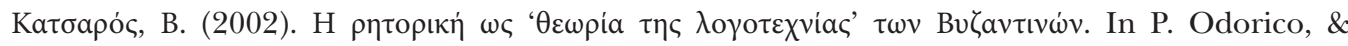
P. A. Agapitos (Eds.), Pour une «nouvelle» histoire de la littérature byzantine. Problèmes, méthodes, approches, propositions. Actes du colloque international philologique Nicosie, 25-28 mai 2000 (pp. 95-106). Paris: Centre d'Études Byzantines, Néo-Helléniques et Sud-Est Européennes.

Kazhdan, P. (1991). The Oxford Dictionary of Byzantium (Vols. I-III). New York - Oxford: Oxford University Press.

Kiourtzian, G. (2005). Un nouveau diptyque en ivoire de style byzantin. In C. Balmelle, P. Chevalier, \& G. Ripoll (Eds.), Mélanges d'antiquité tardive. Studiola in honorem Noël Duval (Bibliothèque de l'antiquité tardive, 5 ; pp. 233-244). Turnhout: Brepols.

Klein, H. A. (2004). Byzanz, der Westen und das „wahre“ Kreuz. Die Geschichte einer Reliquie und ihrer künstlerischen Fassung in Byzanz und im Abendland (Spätantike - Frühes Christentum - Byzanz. Kunst im ersten Jahrtausend. Wiesbaden: Reihe B: Studien und Perspektiven 17.

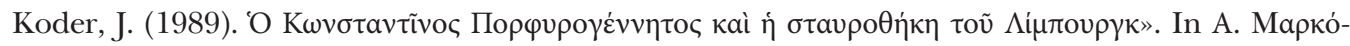

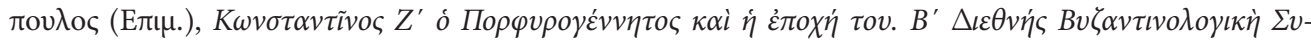

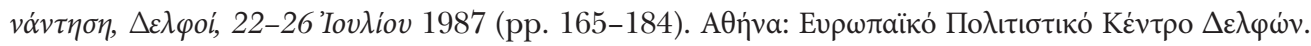

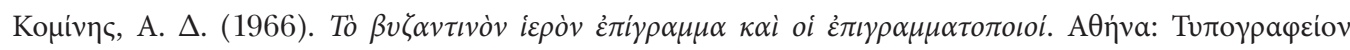

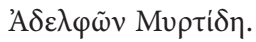

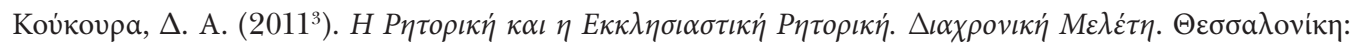

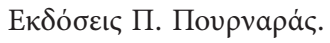

Lagarde, P., \& Bollig, J. (1882=1979). Iohannis Euchaitorum metropolitae quae in Codice Vaticano Graeco 676 supersunt (Abhandlungen der historisch-philologischen Classe der königlichen Gesellschaft der Wissenschaften zu Göttingen, 28). Gottingae: Dietrich (= Amsterdam: Hakkert).

Lauxtermann, M. D. (1994). The Byzantine Epigram in the Ninth and Tenth Centuries. A Generic Study of Epigrams and some other Forms of Poetry. Diss. Amsterdam.

Lauxtermann, M. D. (1998). The velocity of pure iambs. Byzantine observations on the metre and rhythm of the dodecasyllable. Jahrbuch der Österreichischen Byzantinistik 48, 9-33.

Lauxtermann, M. D. (1999). The spring of Rhythm. An Essay on the Political Vers and Other Byzantine Metres. Wien: BV XXII.

Lauxtermann, M. D. (2003a). Byzantine Poetry from Pisides to Geometres. Texts and Contexts. Vol. I. Wien: WBS XXIV/1.

Lauxtermann, M. D. (2003b). Some remarks on Pisides' epigrams and shorter poems. In Hörandner, W., \& M. Grünbart (Eds.). (2003). L'épistolographie et la poésie épigrammatique: projets actuels et questions de méthodologie. Actes de la 16e Table ronde organisée par W. Hörandner et M. Grünbart dans le cadre du XXe Congrès international des Études byzantines, Collège de France - Sorbonne, Paris, 19-25 Août 2001 (pp. 177-189). Paris: Dossiers byzantins 3. 
Longo, A. A., \& Jacob, A. (1980-1982). Une anthologie salentine du XIV siècle: le Vaticanus gr. 1276. Rivista di Studi Bizantini e Neoellenici, 17-19, 149-228.

Maas, P. (1903). Der byzantinische Zwölfsilber. Byzantinische Zeitschrift, 12, 278-323.

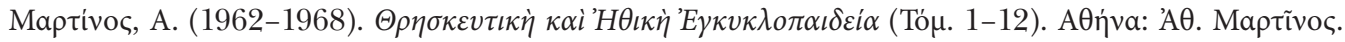
Migne, J.-P. (Ed.). (1857-1866). Patrologiae cursus completus. Series Graeca (Vols. 1-161). Paris: Migne. Miller, M. (1855-1857=1967). Manuelis Philae Carmina (Vol. I/II). Paris: Typograph. Imperiali (= Amsterdam : Hakkert).

Miller, M. (1883). Poésies inédites de Théodore Prodrome. Annuaire de l'Association pour l'encouragement des études grecques en France, 17, 18-64.

Odorico, P., \& Agapitos, A. (Eds.). (2002). Pour une «nouvelle» histoire de la littérature byzantine. Problèmes, méthodes, approches, propositions. Actes du colloque international philologique Nicosie, 25-28 mai 2000. Paris: Centre d'Études Byzantines, Néo-Helléniques et Sud-Est Européennes.

Opstall, E. (2008). Jean Géomètre. Poèmes en hexamètres et en distiques élégiaques. Edition, traduction, commentaire (The Medieval Mediterranean, Peoples, Economies and Cultures, 400-1500, 75). Leiden - Boston: Brill.

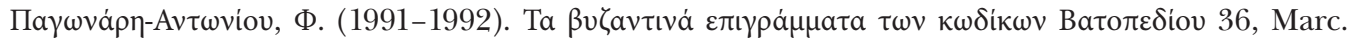

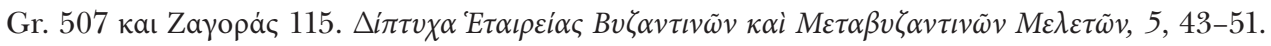

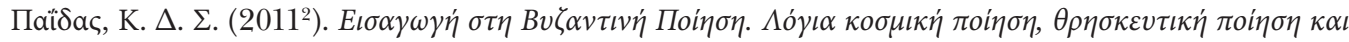

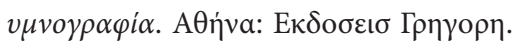

Papagiannis, G. (1997). Theodoros Prodromos. Jambische und hexametrische Tetrasticha auf die Haupterzählungen des Alten und des Neuen Testaments. Einleitung, kritischer Text, Indices (Meletemata, 7; Vol. I/II). Wiesbaden: Beerenverlag.

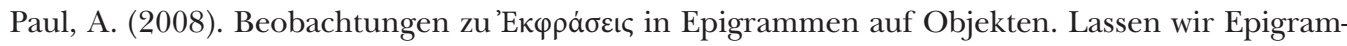
me sprechen! In W. Hörandner, \& A. Rhoby (Eds.), Die kulturhistorische Bedeutung byzantinischer Epigramme. Akten des internationalen Workshops (Wien, 1.-2. Dezember 2006) (Veröffentlichungen zur Byzanzforschung, XIV; pp. 61-73). Wien: Verlag der Österreichischen Akademie der Wissenschaften.

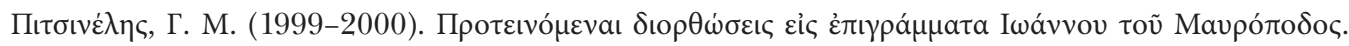

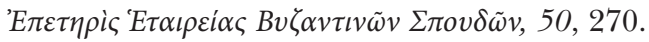

Rhoby, A. (2009). Byzantinische Epigramme auf Fresken und Mosaiken (Veröffentlichungen zur Byzanzforschung, XV; Byzantinische Epigramme in inschriftlicher Überlieferung, ed. W. Hörandner, A. Rhoby, \& A. Paul, Bd. 1). Wien: Verlag der Österreichischen Akademie der Wissenschaften.

Rhoby, A. (2010). Byzantinische Epigramme auf Ikonen und Objekten der Kleinkunst (Veröffentlichungen zur Byzanzforschung, XXIII; Byzantinische Epigramme in inschriftlicher Überlieferung, ed. W. Hörandner, A. Rhoby, \& A. Paul, Bd. 2). Wien: Verlag der Österreichischen Akademie der Wissenschaften.

Rhoby, A. (2011). Inscriptional Poetry - Ekphrasis in Byzantine Tomb Epigrams. Byzantinoslavica 69/3 supplementum, 193-204.

Rhoby, A. (2014). Byzantinische Epigramme auf Stein nebst Addenda zu den Bänden 1 und 2. (Veröffentlichungen zur Byzanzforschung, 35; Byzantinische Epigramme in inschriftlicher Überlieferung, eds. W. Hörandner, A. Rhoby, \& A. Paul, Bd. 3). Wien: Verlag der Österreichischen Akademie der Wissenschaften. 
Rhoby, A. (2018). Byzantinische Epigramme in illuminierten Handschriften (Veröffentlichungen zur Byzanzforschung, XLII; Byzantinische Epigramme in inschrifticher Überlielieferung, ed. W. Hörandner, A. Rhoby, \& A. Paul, Bd. 4). Wien: Verlag der Österreichischen Akademie der Wissenschaften.

Romano, R. (Ed.). (1980). Nicola Callicle, Carmi. Testo critico, introduzione, traduzione, commentario e lessico (Byzantina et Neo-Hellenica Neapolitana, VIII). Neapel: Bibliopolis.

Rott, H. (1908). Kleinasiatische Denkmäler aus Pisidien, Pamphylien, Kappadokien und Lydien. Leipzig: Dieterich.

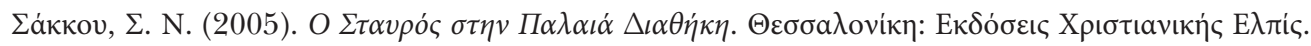

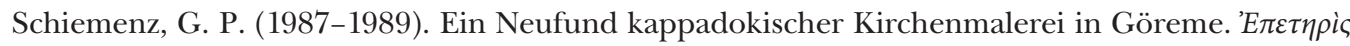

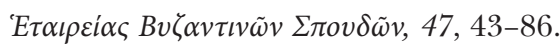

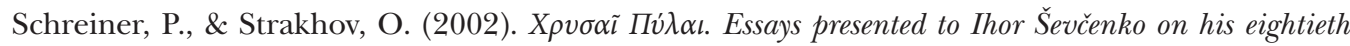
birthday by his colleagues and students (Vols. I-II; Paleoslavica, 10/1-2, 2002). Cambridge, Mass.

Sternbach, I. (1904). Nicolai Calliclis carmina. Rozprawy Akademii Umiejętności. Wydziat filologiczny, ser. II, 21, 315-392.

Sola, J. N. (1911). De Codice Laurentiano X plutei V. Byzantinische Zeitschrift, 20, 373-383.

Thierry, N. (2002). La Cappadoce de l'antiquité au moyen âge (Bibliothèque de l'antiquité tardive, 4). Turnhout: Brepols.

Thierry, N., \& Thierry, M. (1963). Nouvelles églises rupestres de Cappadoce. Région du Hasan Dağı. Nez rock-cut churches of Cappadocia. Avant-propos par André Grabar. Paris: Klincksieck.

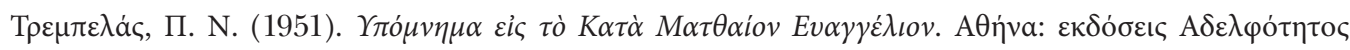

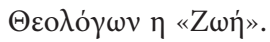

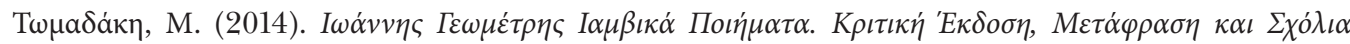

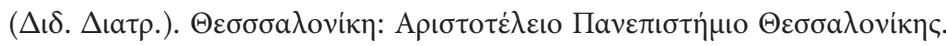

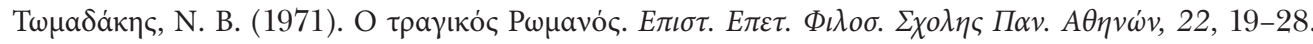

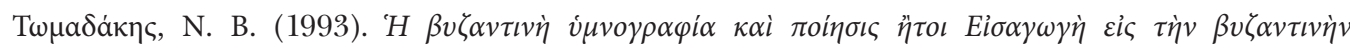

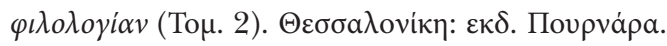

Anastasios Kantaras / kantarastasos@hotmail.com

School of Philosophy - Medieval Greek Philology

Aristotle University of Thessaloniki

Olympou str 122, PO. 546 35, Thessaloniki, Greece

Toto dílo Ize užít v souladu s licenčními podmínkami Creative Commons BY-SA 4 .0 International (https://creativecommons.org/licenses/by-sa/4.0/legalcode). Uvedené se nevztahuje na díla či prvky (např. obrazovou či fotografickou dokumentaci), které jsou v díle užity na základě smluvní licence nebo výjimky či omezení příslušných práv. 
